\title{
Structured Codes Improve the Bennett-Brassard-84 Quantum Key Rate
}

\author{
Graeme Smith, ${ }^{1,2}$ Joseph M. Renes, ${ }^{3}$ and John A. Smolin ${ }^{2}$ \\ ${ }^{1}$ Institute for Quantum Information, California Institute of Technology 107-81, Pasadena, California 91125, USA \\ ${ }^{2}$ IBM T. J. Watson Research Center, Yorktown Heights, New York 10598, USA \\ ${ }^{3}$ Institut für Angewandte Physik, Technische Universität Darmstadt, 64289 Darmstadt, Germany
}

(Received 11 December 2006; published 28 April 2008)

\begin{abstract}
A central goal in information theory and cryptography is finding simple characterizations of optimal communication rates under various restrictions and security requirements. Ideally, the optimal key rate for a quantum key distribution (QKD) protocol would be given by a single-letter formula involving optimization over a single use of an effective channel. We explore the possibility of such a formula for the simplest and most widely used QKD protocol, Bennnett-Brassard-84 with one-way classical postprocessing. We show that a conjectured single-letter formula is false, uncovering a deep ignorance about good private codes and exposing unfortunate complications in the theory of QKD. These complications are not without benefit - with added complexity comes better key rates than previously thought possible. The threshold for secure key generation improves from a bit error rate of 0.124 to 0.129 .
\end{abstract}

DOI: 10.1103/PhysRevLett.100.170502

PACS numbers: 03.67.Dd, 03.67.Hk

Quantum key distribution (QKD) allows two parties using public channels to remotely establish a secret key whose security is not predicated on the difficulty of some computational task. Rather, the security of the key generated by a QKD protocol depends only on fundamental laws of physics. As a result there has been an enormous amount of work on practical and theoretical aspects of QKD, and a corresponding rapid progress in both [1].

The first QKD protocol, Bennnett-Brassard-84 (BB84), was proposed by Bennett and Brassard in 1984 [2], and like all QKD schemes, it is based on the tradeoff between information gain and disturbance in quantum mechanics. To establish a bit of raw key, the sender (Alice) encodes a random bit into one of two conjugate bases $(X$ or $Z$ ), chosen at random, and transmits it to a receiver (Bob). Bob measures in either the $X$ or $Z$ basis, also chosen at random. After generating a large number of bits (say, $2 n$ ), Alice and Bob can sift out the bits for which they both chose the same basis by public discussion, leaving roughly $n$ bits.

Alice then randomly permutes her remaining bits and announces the permutation to Bob, after which they perform parameter estimation by comparing a small fraction of their bits to find the error rate of the sifted key. If the fraction $p$ of bits on which they disagree is sufficiently small, they proceed with information reconciliation and privacy amplification to finally arrive at a secret key. The essence of the protocol is that if an eavesdropper Eve, who is assumed to have control of the quantum channel, examines the signals in order to determine the key, she will necessarily cause some disturbance which manifests itself as errors in the sifted key. Thus $p$ also characterizes how much Eve could have learned about the key.

An important property of any QKD protocol is the amount of noise that can be tolerated without compromis- ing the privacy of the resulting key, the amount of noise at which the protocol aborts. The entanglement-based security proof of Shor and Preskill [3] showed that BB84 can be used to generate a private key for detected bit error rates as high as $p \approx 0.11$, basically by showing there exist Calderbank-Shor-Steane (CSS) [4,5] codes correcting noise up to this level. Remarkably, it was recently found $[6,7]$ that this can be improved to $p \approx 0.124$ if Alice adds independent noise to her sifted key before performing the distillation steps, which has been conjectured to be optimal among all one-way key distillation protocols [7]. The key rates of [6] come from evaluating a single-letter key rate for an effective state found by Devetak and Winter in [8], and indeed the 0.124 threshold of [6,7] is the optimal threshold for this single-letter formula [9]. If these rates were optimal among all protocols, it would indicate a single-letter formula for one-way QKD key-rates, providing a dramatic simplification in the theory of quantum key distribution protocols.

We will show that $p \approx 0.124$ is not optimal, and the threshold is at least $p \approx 0.129$. We increase the threshold by finding improved error correcting codes for the information reconciliation phase. The technique is analogous to those of [10-12], which use degenerate CSS codes to achieve higher quantum capacities than are achievable by the single-letter formula for quantum capacity arising from random stabilizer codes. Though the true maximization needed for the multiletter capacity formula in [8] remains out of reach, we are able to evaluate rates for particular multiletter inputs which achieve higher key rates than the single-letter maximum. While this is suggestive, we emphasize that our results to not necessarily rule out a singleletter formula for the one-way key rate. We have shown that the single-letter Devetak-Winter formula does not give the one-way distillable key, but this does not preclude the 
existence of some other single-letter optimization problem that gives the optimal key rate.

Taken together, our information reconciliation and privacy amplification steps can be described by a highly degenerate CSS code. A quantum code is called degenerate if its syndrome does not uniquely identify the errors which it corrects. This is a uniquely quantum effect-there is no such thing as a degenerate classical code-and all such codes involve entanglement. It appears remarkable then that degeneracy should help in the classical processing task of key distillation. Moreover, Alice and Bob need not perform any multiparticle quantum operations even in our improved protocol. The resolution is that Eve's best attacks involve entanglement, and degeneracy will make this work against her.

Degenerate codes have been used for QKD before, specifically, to improve the threshold of the six-state protocol from 0.126 to 0.127 [13]. However, this protocol did not involve noisy processing, and in fact a better threshold was obtained for the six-state protocol by [6,7]. Our result combines degenerate codes with noisy processing, leading to an advantage over either one alone.

Analytic key-rate expression. - To determine the secret key rate of the modified protocol, we follow $[6,7,14]$. First, the prepare and measure protocol can be converted to an equivalent scheme in which Alice prepares the maximally entangled state $\left|\Phi^{+}\right\rangle_{A B}^{\otimes m n}$ and sends half to Bob. Each party then randomly and independently measures either $X$ or $Z$ on each signal, saving the outcomes for use in parameter estimation and key generation. They discard the outcomes where their basis choice did not agree, and denoting the remaining outcomes $K_{A}$ and $K_{B}$ it follows from Corollary 6.5.2 of [14] that for any $m$-bit processing step $K_{A}^{m} \rightarrow U$ and $U \rightarrow V$ it is possible to use standard (i.e., unstructured, random) error correction and privacy amplification to distill a secret key at rate

$$
r=\frac{1}{m} \inf _{\sigma_{A B} \in \Gamma_{p}}\left[S\left(U \mid V E^{m}\right)-S\left(U \mid V K_{B}^{m}\right)\right],
$$

evaluated on the state generated by performing the processing on $\sigma_{A B}^{\otimes m}$, and where $\Gamma_{p}$ is the set of single pair Belldiagonal states $\sigma_{A B}$ passing the parameter estimation phase of the protocol and $E^{m}$ is the purification of $\sigma_{A B}^{\otimes m}$, which we must assume belongs to Eve. $S(\rho)=-\operatorname{Tr} \rho \log \rho$ is the von Neumann entropy. This expression is similar to what was found in [6,7], with the additional feature that it includes blockwise processing. Since the $X$ and $Z$ bases are randomly used to create the sifted key, the error estimation provides an estimate of the bit- and phase-flip noise rates, so that the allowable $\sigma_{A B}$ are of the form $\sigma_{A B}=$ $(1+t-2 p)\left|\Phi^{+}\right\rangle\left\langle\Phi^{+}\right|+(p-t)\left(\left|\Phi^{-}\right\rangle\left\langle\Phi^{-}|+| \Psi^{+}\right\rangle \times\right.$ $\left.\left\langle\Psi^{+}\right|\right)+t\left|\Psi^{-}\right\rangle\left\langle\Psi^{-}\right|$for $t \in[0, p]$.

Below, we choose a particular $K_{A}^{m} \rightarrow U \rightarrow V$ for which Eq. (1) outperforms all previously known protocols for large $p$. The measurements leading to $K_{A}$ and $K_{B}$ will be the same as for the usual BB84 protocol, with the processing step chosen as follows. For each $m$-bit block of $K_{A}$ $\left(x_{1}, x_{2}, \ldots, x_{m}\right)$ Alice independently flips each bit with probability $q$, resulting in $\tilde{\mathbf{x}}=\left(\tilde{x}_{1}, \ldots, \tilde{x}_{m}\right)$. She then computes $U=\left(\tilde{x}_{1}, \tilde{x}_{1} \oplus \tilde{x}_{2}, \ldots, \tilde{x}_{1} \oplus \tilde{x}_{m}\right)$ and sends $V=\left(\tilde{x}_{1} \oplus\right.$ $\left.\tilde{x}_{2}, \ldots, \tilde{x}_{1} \oplus \tilde{x}_{m}\right)$ to Bob, after which they do error correction and privacy amplification as usual. The key rate they achieve is given by the following theorem.

Theorem 1.- The key rate achieved using the processing $\mathbf{x} \rightarrow U \rightarrow V$ with $U=\left(\tilde{x}_{1}, \tilde{x}_{1} \oplus \tilde{x}_{2}, \ldots, \tilde{x}_{1} \oplus \tilde{x}_{m}\right), \quad V=$ $\left(\tilde{x}_{1} \oplus \tilde{x}_{2}, \ldots, \tilde{x}_{1} \oplus \tilde{x}_{m}\right)$, where $\tilde{\mathbf{x}}=\mathbf{x} \oplus \mathbf{f}$ and $\mathbf{f}$ is a string of independent 0-1 random variables, each with probability $q$ of being 1 , is given by

$$
\begin{aligned}
r= & \frac{1}{m}\left[1-\sum_{\mathbf{s}} P_{m}^{\tilde{p}}(\mathbf{s}) H\left(P_{m}^{\tilde{p}}(u \mid \mathbf{s})\right)+m S\left(\rho_{p, q}\right)\right. \\
& \left.-S\left(\frac{1}{2} \rho_{p, q}^{\otimes m}+\frac{1}{2} Z^{\otimes m} \rho_{p, q}^{\otimes m} Z^{\otimes m}\right)\right] .
\end{aligned}
$$

Here $\rho_{p, q}=(1-q)\left|\varphi_{+}\right\rangle\left\langle\varphi_{+}|+q| \varphi_{-}\right\rangle\left\langle\varphi_{-}\right|$with $\left|\varphi_{ \pm}\right\rangle=$ $\sqrt{1-p}|0\rangle \pm \sqrt{p}|1\rangle, \quad \tilde{p}=p(1-q)+q(1-p), \quad$ while $P_{m}^{\tilde{p}}(u, \mathbf{s})$ is defined in Lemma 2. The entropy $H$ of a classical probability distribution $P$ is given by $H(P)=$ $-\sum_{l} P_{l} \log P_{l}$.

We proceed by noting that in the entanglement picture, our processing step is equivalent to Alice first adding independent bit errors to her halves of the noisy EPR pairs, measuring the stabilizers of an $m$-qubit repetition code, and then sending her syndrome outcomes to Bob. We apply the following lemma, which follows from [12].

Lemma 2. - The $m$-qubit repetition code with stabilizers $Z_{1} Z_{2}, \ldots, Z_{1} Z_{m}$ maps the error $X^{\mathbf{u}} Z^{\mathbf{v}}$ to the logical error $X^{u_{1}} Z^{\Theta_{l=1}^{m} v_{l}}$ and syndrome $\mathbf{s}=\left(u_{1} \oplus u_{2}, \ldots, u_{1} \oplus u_{m}\right)$. When used to correct independent bit errors of probability $p$, the probability of a logical bit error $u$ and syndrome $\mathbf{s}$ is given by

$$
P_{m}^{p}(u, \mathbf{s})=\left[p^{m-s}(1-p)^{s}\right]^{u}\left[p^{s}(1-p)^{m-s}\right]^{1-u}
$$

for $s=|\mathbf{s}|$.

Proof of Theorem 1.-To evaluate Eq. (1), first let

$$
\sigma_{A B}^{\otimes m}=\sum_{\mathbf{u}, \mathbf{v}} p_{\mathbf{u v}} X_{B}^{\mathbf{u}} Z_{B}^{\mathbf{v}}\left[\left|\Phi^{+}\right\rangle\left\langle\Phi^{+}\right|\right]_{A B}^{\otimes m} Z_{B}^{\mathbf{v}} X_{B}^{\mathbf{u}},
$$

with $p_{\mathbf{u v}}$ such that $p_{\mathbf{u}}=\sum_{\mathbf{v}} p_{\mathbf{u v}}=p^{|\mathbf{u}|}(1-p)^{m-|\mathbf{u}|}$, for measured bit error rate $p$, and similarly for $p_{\mathbf{v}}$.

Alice adds independent noise at error rate $q$ to the $A$ register, so the state of the Alice-Bob-Eve system can be described as

$$
\sum_{\mathbf{u}, \mathbf{v}, \mathbf{f}} \sqrt{p_{\mathbf{u v}} q_{\mathbf{f}}}|\mathbf{f}\rangle_{A^{\prime}} X_{B}^{\mathbf{u}} Z_{B}^{\mathbf{v}} X_{B}^{\mathbf{f}}\left|\Phi^{+}\right\rangle_{A B}^{\otimes m}|\mathbf{u}\rangle_{E_{1}}|\mathbf{v}\rangle_{E_{2}}
$$

where we have used the fact that $X_{A} \otimes I\left|\Phi^{+}\right\rangle_{A B}=$ $I \otimes X_{B}\left|\Phi^{+}\right\rangle_{A B}$. Note that Eve's system is determined by the fact that in the worst case she holds the purification of 
the state after it emerges from the channel. However, she does not hold the purification of the noise Alice adds.

Alice and Bob then measure the stabilizers of the $m$-qubit repetition code $\left(Z_{1} Z_{2}, \ldots, Z_{1} Z_{m}\right)$ and Alice sends her outcomes to Bob. This is equivalent to having Bob defer his measurement until he receives Alice's message and then coherently correcting his key bit, which we will consider here. Renaming Bob's $m-1$ syndrome qubits system $B^{\prime}$, the state they will share in this case is

$$
\begin{aligned}
& \sum_{\mathbf{u}, \mathbf{v}, \mathbf{f}} \sqrt{p_{\mathbf{u v}} q_{\mathbf{f}}}|\mathbf{f}\rangle_{A^{\prime}} X_{B}^{u_{1} \oplus f_{1}} Z_{B}^{\oplus_{l=1}^{m} v_{l}}\left|\Phi^{+}\right\rangle_{A B} \\
& \quad \otimes\left|\mathbf{s}_{\mathbf{u}, \mathbf{f}}\right\rangle_{B^{\prime}}|\mathbf{u}\rangle_{E_{1}} Z_{E_{2}}^{\mathbf{f}}|\mathbf{v}\rangle_{E_{2}},
\end{aligned}
$$

where $\mathbf{s}_{\mathbf{u}, \mathbf{f}}$ is an $(m-1)$-bit string labeling the basis states of $B^{\prime}$ whose $j$ th bit is $\left(\mathbf{s}_{\mathbf{u}, \mathbf{f}}\right)_{j}=u_{1} \oplus u_{j+1} \oplus f_{1} \oplus f_{j+1}$. Note that the $Z^{\mathbf{f}}$ acting on Eve's second system comes from the commutation of $Z_{B}^{\mathbf{v}}$ and $X_{B}^{\mathbf{f}}$.

Getting rid of the $A^{\prime}$ system (but keeping it from Eve), we now let Alice and Bob measure systems $A$ and $B B^{\prime}$ in the computational basis, respectively. According to Eq. (1), the difference of conditional entropies for the resulting state will give us the key rate. This will be simpler to analyze by first rewriting the lower bound as

$$
r \geq \frac{1}{m} \inf _{\sigma_{A B} \in \Gamma_{p}} I\left(A ; B B^{\prime}\right)-I(A ; E) .
$$

$I\left(A ; B B^{\prime}\right)$ is the mutual information $[I(X ; Y)=$ $S(X)+S(Y)-S(X Y)]$ of $\rho_{A B B^{\prime}}=\frac{1}{2} \sum_{x=0}^{1}|x\rangle\left\langle\left. x\right|_{A} \otimes \rho_{B^{\prime} B}^{x}\right.$, where

$$
\begin{aligned}
\rho_{B^{\prime} B}^{x}= & \sum_{\mathbf{f}} \sum_{\mathbf{u}} q_{\mathbf{f}} p_{\mathbf{u}}\left|x+f_{1}+u_{1}\right\rangle\left\langle x+f_{1}+\left.u_{1}\right|_{B} \otimes \mid \mathbf{s}_{\mathbf{u}, \mathbf{f}}\right\rangle \\
& \times\left\langle\mathbf{s}_{\mathbf{u}, \mathbf{f}}\right| \\
= & \sum_{\mathbf{s}} P_{m}^{\tilde{p}}(\mathbf{s}) \sum_{u=0}^{1} P_{m}^{\tilde{p}}(u \mid \mathbf{s})|x+u\rangle\left\langle x+\left.u\right|_{B} \otimes \mid \mathbf{s}\right\rangle\left\langle\left.\mathbf{s}\right|_{B^{\prime}},\right.
\end{aligned}
$$

and the $P_{m}^{\tilde{p}}(u, \mathbf{s})$ are given by Lemma 2 . Thus, the mutual information $I\left(A ; B B^{\prime}\right)$ is exactly $1-\sum_{\mathbf{s}} P_{m}^{\tilde{p}}(\mathbf{s}) H\left(P_{m}^{\tilde{p}}(u \mid \mathbf{s})\right)$. Notice that this term only depends on $p_{\mathbf{u}}$, which is determined by the parameter estimation phase, so it will be the same for all $\sigma_{A B} \in \Gamma_{p}$.

Turning to the second term in Eq. (7), we want to find the mutual information of the Alice-Eve system, $\rho_{A E_{1} E_{2}}=\frac{1}{2} \times$ $\sum_{x=0}^{1}|x\rangle\left\langle\left. x\right|_{A} \otimes \rho_{E_{1} E_{2}}^{x}\right.$, where

$$
\begin{aligned}
\rho_{E_{1} E_{2}}^{x}= & \left(Z_{E_{2}}^{\otimes m}\right)^{x}\left(\sum _ { \mathbf { u } , \mathbf { v } _ { 1 } , \mathbf { v } _ { 2 } , \mathbf { f } } q _ { \mathbf { f } } \sqrt { p _ { \mathbf { u } | \mathbf { v } _ { 1 } } p _ { \mathbf { u } | \mathbf { v } _ { 2 } } } | \mathbf { u } \rangle \left\langle\left.\mathbf{u}\right|_{E_{1}}\right.\right. \\
& \otimes \sqrt{p_{\mathbf{v}_{1}} \bar{p}_{\mathbf{v}_{2}}} Z^{\mathbf{f}}\left|\mathbf{v}_{1}\right\rangle\left\langle\left.\mathbf{v}_{2}\right|_{E_{2}} Z^{\mathbf{f}}\right)\left(Z_{E_{2}}^{\otimes m}\right)^{x} .
\end{aligned}
$$

Note that the $\left(Z_{E_{2}}^{\otimes m}\right)^{x}$ comes from the action of $Z^{\oplus_{l=1}^{m} v_{l}}$ on $B$. When bit and phase errors are independent, this expression can be further simplified. Defining $\mu=\sum_{\mathbf{u}} p_{\mathbf{u}}|\mathbf{u}\rangle\langle\mathbf{u}|$ and $\rho_{p, q}=(1-q)\left|\varphi_{+}\right\rangle\left\langle\varphi_{+}|+q| \varphi_{-}\right\rangle\left\langle\varphi_{-}\right| \quad$ with $\left|\varphi_{ \pm}\right\rangle=$ $\sqrt{1-p}|0\rangle \pm \sqrt{p}|1\rangle$, we can write

$$
\rho_{E_{1}, E_{2}}^{x}=\mu_{E_{1}} \otimes\left(Z_{E_{2}}^{\otimes m}\right)^{x}\left[\rho_{p, q}^{\otimes m}\right]_{E_{2}}\left(Z_{E_{2}}^{\otimes m}\right)^{x} .
$$

Actually, we have to maximize $I\left(A ; E_{1} E_{2}\right)$ overall $p_{\mathbf{u v}}$ corresponding to states in $\sigma_{A B} \in \Gamma_{p}$, but the largest value is attained for independent phase and bit errors. This means that Eve's optimal attack on the protocol will be to choose $\sigma_{A B} \in \Gamma_{p}$ with $t=p^{2}$. In particular, if Eve starts with the independent $\mathbf{u}, \mathbf{v}$ state, by tracing out the $E_{1}$ system and using the isometry

$$
U=\sum_{\mathbf{v}, \mathbf{u}} \sqrt{p_{\mathbf{u} \mid \mathbf{v}}}|\mathbf{u}\rangle_{E_{3}}|\mathbf{v}\rangle_{E_{2}}\left\langle\left.\mathbf{v}\right|_{E_{2}},\right.
$$

then completely dephasing $E_{3}$, she can construct a $\rho_{A E_{2} E_{3}}$ with the same mutual information as if the errors were distributed according to $p_{\mathbf{u} \mid \mathbf{v}} p_{\mathbf{v}}$. Since mutual information cannot be increased by local operations, the independent noise state must have the largest value. Moreover, as the $E_{1}$ system is uncorrelated with $A E_{2}, I(A ; E)$ can be easily computed, yielding

$$
I(A ; E)=S\left(\frac{1}{2} \rho_{p, q}^{\otimes m}+\frac{1}{2} Z^{\otimes m} \rho_{p, q}^{\otimes m} Z^{\otimes m}\right)-m S\left(\rho_{p, q}\right) .
$$

Taking the difference between $I\left(A ; B B^{\prime}\right)$ and $I(A ; E)$, keeping in mind we must send $m$ qubits for each $m$ block, leads to the overall key rate of Eq. (2).

Numerical key rates. - We now evaluate Eq. (2) for particular $p, q$, and $m . S\left(\rho_{p, q}\right)$ is easily calculated, and the second term can be evaluated efficiently via Eq. (3). The most difficult term is $S\left(\frac{1}{2} \rho_{p, q}^{\otimes m}+\frac{1}{2} Z^{\otimes m} \rho_{p, q}^{\otimes m} Z^{\otimes m}\right)$, but it can be handled as follows. Because of the permutation invariance of the state $\rho_{p, q}^{\otimes m}$, it is compactly expressed as a direct sum over the $S U(2)$ irreducible representations (irreps). Each irrep occurs with some degeneracy, giving a permutation factor, which by Schur's lemma [15] is maximally mixed. Using the expression for multiple copies of a qubit mixed state from [16], which gives the irreducible states of $\rho_{p, q}^{\otimes m}$ as a function of its Bloch vector and doing the same for $Z^{\otimes m} \rho_{p, q}^{\otimes m} Z^{\otimes m}$, we can compute $S\left(\frac{1}{2} \rho_{p, q}^{\otimes m}+\frac{1}{2} Z^{\otimes m} \rho_{p, q}^{\otimes m} Z^{\otimes m}\right)$ for $m$ up to several hundred.

In general, larger $m$ gives higher thresholds with the optimal $q \approx 0.3$ increasing slowly with $m$ (Fig. 1). $m=$ 400 and $q=0.32$ give nonzero key rate up to $p=0.1292$, but for larger $m$ the computation becomes quite slow.

Discussion. - Given the pattern of improving thresholds with larger $m$, it is tempting to guess the best threshold within our family of codes will be when $m \rightarrow \infty$ as $q \rightarrow$ 0.5 . While we have not been able to do so, we hope that an asymptotic analysis of our key rates in the limit of large $m$ could be tractable. Along these lines, note that an exact analysis of large repetition codes in the context of quantum capacities was successfully carried out in [10].

We note that our codes are highly restricted, and it is not at all clear that they should be optimal. One idea for better 


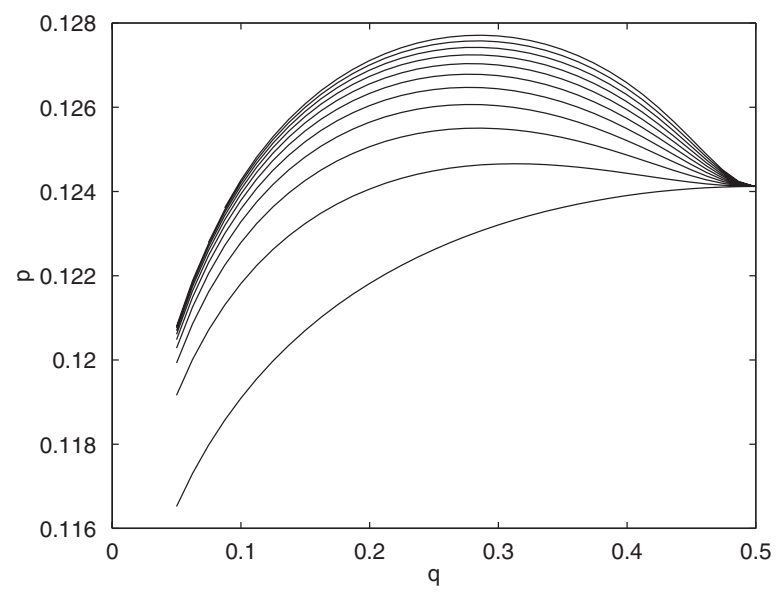

FIG. 1. Bit error rate $p$ at which the key rate goes to zero as a function of processing noise $q$ when using various-sized repetition codes in the BB84 protocol. The curves are, from bottom to top, $m=1, m=10,20, \ldots, 100$, illustrating the fact that a longer repetition code allows a higher threshold. As $m$ is increased, the optimal $q$ also grows. Taking $m=400$ and $q=$ 0.32 gives our best threshold of 0.1292 .

rates is to adapt the concatenation of repetition codes in conjugate bases used in $[11,12]$ to key generation, using a repetition code in the $X$ basis to improve privacy amplification. A more ambitious approach is to develop new degenerate codes for this problem, perhaps using the heuristic suggested in [12].

The best upper bound on the BB84 key rate is $H(1 / 2-$ $2 p(1-p))-H(2 p(1-p))$ [17]. This gives an upper bound on the threshold for BB84 of $p=(1-1 / \sqrt{2}) / 2 \approx$ 0.1464 , matching the bound due to the optimal individual attack found in [18]. There remains a significant gap between our lower bound of 0.129 and this upper bound.

Our one-way protocols bear a striking resemblance to two-way protocols using advantage distillation [19]. In particular, an advantage distillation protocol can be described as using a repetition code, with Bob sending the syndromes back to Alice. Error correction and privacy amplification are performed on blocks for which no error is detected, while the blocks for which an error is detected are thrown away. Without back communication from Bob, Alice would not know the syndromes, and thus be unable to discard blocks in which Bob had detected an error. Our findings show that even in this case, with Alice ignorant of the syndromes, and thus unable to discard bad blocks, there is still a benefit in using a repetition code. The repetition code works "better than expected," because it collapses many phase errors to a single logical phase error, while still providing information about bit errors. This benefit should also appear when the code is used for advantage distillation with noisy processing.
One-way protocols with noisy processing can be viewed quite naturally as distillation protocols for twisted EPR pairs [20,21]. In [20] it was shown that noisy processing can be interpreted as the deflection of Eve's correlations away from the sifted key into a "shield" system, which purifies the noise added by Alice. Viewed in this way, the benefit of a repetition code is that it allows us to combine the "soft" approach of deflecting phase errors and the "hard" approach of correcting bit errors - while learning about bit errors that we must correct, we are simultaneously decreasing Eve's correlation with the key, reducing the need for privacy amplification later.

We thank Debbie Leung, John Preskill, and Renato Renner for several valuable discussions. This work grew out of discussions between G.S. and J.M.R. at the University of Queensland, whose hospitality we appreciate. J.M.R. acknowledges the Alexander von Humboldt Foundation, G.S. NSF Grant No. PHY-0456720 and Canada's NSERC, and J.A.S. ARO Contract No. DAAD19-01-C-0056.

[1] N. Gisin et al., Rev. Mod. Phys. 74, 145 (2002).

[2] C. H. Bennett and G. Brassard, in Proceedings of the IEEE International Conference on Computers, Systems, and Signal Processing (IEEE, Bangalore, India, 1984), p. 175.

[3] P. W. Shor and J. Preskill, Phys. Rev. Lett. 85, 441 (2000).

[4] A. Steane, Proc. R. Soc. A 452, 2551 (1996).

[5] A. R. Calderbank and P. W. Shor, Phys. Rev. A 54, 1098 (1996).

[6] B. Kraus et al., Phys. Rev. Lett. 95, 080501 (2005).

[7] R. Renner et al., Phys. Rev. A 72, 012332 (2005).

[8] I. Devetak and A. Winter, Proc. R. Soc. A 461, 207 (2005).

[9] The optimality of the 0.124 threshold for the single-letter Devetak-Winter formula has not been proven in the literature, but is easily verified by numerical optimization.

[10] P. W. Shor and J. A. Smolin, arXiv:quant-ph/9604006.

[11] D. P. DiVincenzo et al., Phys. Rev. A 57, 830 (1998).

[12] G. Smith and J. A. Smolin, Phys. Rev. Lett. 98, 030501 (2007).

[13] H.-K. Lo, Quantum Inf. Comput. 1, 81 (2001).

[14] R. Renner, Ph.D. thesis, ETH, 2005.

[15] B. Simon, Representations of Finite and Compact Groups (AMS, USA, 1996).

[16] E. Bagan et al., Phys. Rev. A 73, 032301 (2006).

[17] G. Smith and J. A. Smolin, arXiv:0712.2471.

[18] C. A. Fuchs et al., Phys. Rev. A 56, 1163 (1997).

[19] D. Gottesman and H.-K. Lo, IEEE Trans. Inf. Theory 49, 457 (2003).

[20] J. M. Renes and G. Smith, Phys. Rev. Lett. 98, 020502 (2007).

[21] K. Horodecki et al., Phys. Rev. Lett. 94, 160502 (2005). 